\title{
ON THE DUAL WEIGHTS FOR CROSSED PRODUCTS OF VON NEUMANN ALGEBRAS II
} Application of operator valued weights

\section{UFFE HAAGERUP}

\section{Introduction.}

Let $M \otimes_{\alpha} G$ be the crossed product of a von Neumann algebra and a locally compact group $G$, acting on $M$. The von Neumann algebra $M \otimes_{\alpha} G$ is generated by $\pi(M)$ and $\lambda(G)$ for a certain covariant representation $(\pi, \lambda)$ of $(M, G, \alpha)$. We prove that there is a unique normal faithful semifinite (n.f.s.) operator valued weight $T$ from $M \otimes_{\alpha} G$ to $\pi(M)$, such that for any n.f.s. weight $\varphi$ on $M$, the dual weight $\tilde{\varphi}$ on $M \otimes_{\alpha} G$ is given by $\tilde{\varphi}=\left(\varphi \circ \pi^{-1}\right) \circ T$. When $G$ is abelian, $T$ is given by the formula

$$
T x=\int_{\hat{G}} \hat{\alpha}_{p}(x) d p, \quad x \in\left(M \otimes_{\alpha} G\right)_{+}
$$

where $\hat{\alpha}$ is the dual action on $M \otimes_{\alpha} G$. When $G$ is discrete, $T$ is the positive part of the normal conditional expectation $\varepsilon$ from $M \otimes_{\alpha} G$ to $\pi(M)$ given by

$$
\varepsilon\left(\sum_{s \in G} \lambda(s) \pi(x(s))=\pi(x(e))\right.
$$

for any $M$-valued function $x$ from $G$ to $M$ with finite support.

Let us recall the main results on operator valued weights (cf. [7]). Let $M$ and $N$ be von Neumann algebras, $N \cong M$. An operator valued weight $T$ from $M$ to $N$ is a map of $M_{+}$into the extended positive part $\hat{N}_{+}$of $N$ (i.e. the set of homogeneous, additive and lower semi-continuous functions on $N_{*}^{+}$with values in $[0, \infty])$ with the properties:

(1) $T(x+y)=T(x)+T(y), \quad x, y \in M_{+}$

(2) $T(\lambda x)=\lambda T(x), \quad x \in M_{+}, \lambda \geqq 0$,

(3) $T\left(a^{*} x a\right)=a^{*} T(x) a, \quad x \in M_{+}, a \in N$.

$T$ is normal if $x_{i}>x \Rightarrow T\left(x_{i}\right)>T(x)$. T is faithful if $T\left(x^{*} x\right)=0 \Rightarrow x=0$ and 
semifinite if the set of $x \in M$ for which $\left\|T\left(x^{*} x\right)\right\|<\infty$ is $\sigma$-strongly dense in $M$. We let $P(R)$ denote the set of n.f.s. weights on a von Neumann algebra $R$, and $P(M, N)$ denotes the set of n.f.s. operator valued weights from $M$ to $N$. Any normal weight $\varphi$ on $N_{+}$has a unique normal extension (also denoted $\varphi$ ) to $\hat{N}_{+}$. If $\varphi \in P(N)$ and $T \in P(M, N)$ then $\varphi \circ T \in P(M)$. Moreover

$$
\begin{array}{ll}
\sigma_{t}^{\varphi \circ T}(x)=\sigma_{t}^{\varphi}(x) & x \in N, \varphi \in P(N) . \\
(D \psi \circ T: D \varphi \circ T)_{t}=(D \psi: D \varphi)_{t} & \varphi, \psi \in P(N) .
\end{array}
$$

(cf. [7, proposition 1.11 and Theorem 4.7]).

Conversely if $\varphi \rightarrow \tilde{\varphi}$ is a map of $P(N)$ into $P(M)$ that satisfies:

$$
\begin{array}{ll}
\sigma_{t}^{\tilde{\varphi}}(x)=\sigma_{t}^{\varphi}(x), & x \in N, \varphi \in P(N) \\
(D \tilde{\psi}: D \tilde{\varphi})_{t}=(D \psi: D \varphi)_{t} & \varphi, \psi \in P(N)
\end{array}
$$

then there is a unique $T \in P(M, N)$, such that $\tilde{\varphi}=\varphi \circ T$ for any $\varphi \in P(N)$, (cf. [7, corollary 5.4]).

In [8] we gave a general construction of the dual weights on the crossed product $M \otimes_{\alpha} G$ of a von Neumann algebra $M$ and a locally compact group $G$. (cf. [4], [11] and [14]). Moreover we proved [8, Theorem 3.2] that

$$
\begin{array}{ll}
\sigma_{t}^{\dot{\varphi}}(\pi(x))=\pi\left(\sigma_{t}^{\varphi}(x)\right) & x \in M, \varphi \in P(M) \\
(D \tilde{\psi}: D \tilde{\varphi})_{t}=\pi\left((D \psi: D \varphi)_{t}\right) & \varphi, \psi \in P(M)
\end{array}
$$

where $\tilde{\varphi}$ and $\psi$ denote the dual weights of $\varphi$ and $\psi$. An easy combination of the above results gives:

There is a unique n.f.s. operator valued weight $T$ from $M \otimes_{\alpha} G$ to $\pi(M)$, such that for any $\varphi \in P(M)$, the dual weight $\tilde{\varphi}$ on $M \otimes_{\alpha} G$ is given by $\tilde{\varphi}=\left(\varphi \circ \pi^{-1}\right) \circ T$.

The main purpose of this paper is to obtain a concrete formula for this operator valued weight $T$. In the case, $G$ abelian, we prove in section 1 that

$$
T x=\int_{\hat{G}} \hat{\alpha}_{p}(x) d p, \quad x \in\left(M \otimes_{\alpha} G\right)_{+}
$$

where $\hat{\alpha}$ is the dual action of $\hat{G}$ on $\left(M \otimes_{\alpha} G\right)_{+}$, and $d p$ is the dual Haar measure. Let now $M \otimes_{\alpha} G$ be a crossed product with an arbitrary locally compact group, and let $P(G)$ denote the set of continuous, positive, definite functions on $G$.

For $\varphi \in P(G)$ we write $\varphi \ll \delta$ if $\varphi$ is less than the Dirac measure in $e$ (unit element) with respect to the ordering of positive definite measures on $G$. In section 3 we will prove: 
(1) For any $\varphi \in P(G)$ there is a unique completely positive, normal linear map $E_{\varphi}$ on $M \otimes_{\alpha} G$ such that

$$
\begin{array}{ll}
E_{\varphi}(a x b)=a E_{\varphi}(x) b, & x \in M \otimes_{\alpha} G, a, b \in \pi(M) \\
E_{\varphi}(\lambda(s))=\varphi(s) \lambda(s), & s \in G
\end{array}
$$

(2) The formula

$$
T x=\sup _{\varphi \ll \delta} E_{\varphi} x, \quad x \in\left(M \otimes_{\alpha} G\right)_{+}
$$

defines a n.f.s. operator valued weight from $M \otimes_{\alpha} G$ to $\pi(M)$, such that $\tilde{\varphi}=$ $\left(\varphi \circ \pi^{-1}\right) \circ T$ for any $\varphi \in P(M)$.

The hard part of the proof is to show that the range of $T$ is contained in the extended positive part of $\pi(M)$. The above construction of the operator valued weight $T$ is inspired by Landstads paper [9]. In fact it is not hard to verify that $T$ is an extension of the "generalized conditional expectation" $P \circ \delta$ in $[9$, lemma $2.8]$. For the proof of (2) we need detailed information about the canonical weight $\Omega$ on the von Neumann algebra $\mathscr{L}(G)$ associated with the left regular representation of $G$ (see for instance $[10, \S 1]$ ). We believe that these results about $\Omega$ are more or less known, but as we have been unable to find them in the literature, we will derive (in section 2) the results needed for our applications.

\section{Crossed products with abelian groups.}

Let $M$ be a von Neumann algebra on a Hilbert space $H$, and let $\alpha$ : $G \rightarrow$ aut $(M)$ be a $\sigma$-weakly continuous action of a locally compact group on $M$. Put

$$
\begin{aligned}
& (\pi(x) \xi)(t)=\alpha_{t}^{-1}(x) \xi(t) \quad x \in M, \xi \in L^{2}(G, H) \\
& (\lambda(s) \xi)(t)=\xi\left(s^{-1} t\right) \quad s \in G, \xi \in L^{2}(G, H) .
\end{aligned}
$$

$(\pi, \lambda)$ is a covariant representation of $(M, G, \alpha)$ i.e.

$$
\pi\left(\alpha_{s} x\right)=\lambda(s) \pi(x) \lambda(s)^{*}, \quad x \in M, s \in G .
$$

The von Neumann algebra on $L^{2}(G, H)$ generated by $\pi(M)$ and $\lambda(G)$ is called the crossed product of $M$ and $G$, and is denoted $M \otimes_{\alpha} G$. (cf. [14, definition 3.1]). We let $K(G, M)$ denote the set of $\sigma$-strong* continuous functions from $G$ to $M$ with compact support. $K(G, M)$ is an involutive algebra with product

$$
(x * y)(s)=\int_{G} \alpha_{t} x(s t) y\left(t^{-1}\right) d t, \quad x, y \in K(G, M)
$$

and involution 


$$
x^{\#}(s)=\Delta_{G}(s)^{-1} \alpha_{s}^{-1} x\left(s^{-1}\right)^{*}, \quad x \in K(G, M)
$$

where $\Delta_{G}(s)$ is the module function on $G$ (cf. [8, lemma 2.3]). Let $(\pi, \lambda)$ be the above covariant representation of $(M, G, \alpha)$. The formula

$$
\mu(x)=\int i(s) \pi(x(s)) d s, \quad x \in K(G, M)
$$

defines a ${ }^{*}$ representation $\mu$ of the involutive algebra $K(G, M)$ on $L^{2}(G, H)$. Moreover $\mu$ maps $K(G, M)$ onto a $\sigma$-weakly dense subset of $M \otimes_{\alpha} G$ ([8, lemma 2.3]).

Assume now that $G$ is abelian, and let $\hat{\alpha}$ be the dual action of $\hat{G}$ on $M \otimes_{x} G$ [12, definition 4.1]. The automorphisms $\hat{\alpha}_{p}, p \in \hat{G}$, satisfy

$$
\begin{array}{ll}
\hat{\alpha}_{p}(\pi(a))=\pi(a), & a \in M, \\
\hat{\alpha}_{p}(\lambda(s))=\overline{\langle p, s\rangle} \lambda(s), & s \in G .
\end{array}
$$

Moreover $\pi(M)$ is exactly the fixpoint algebra of $M \otimes_{\alpha} G$ under $\hat{\alpha}_{p}, p \in \hat{G}$ by $[9, \S 2.5$, Theorem 2], (see also $[8$, lemma 3.6]).

THEOREM 1.1 Let $M \otimes_{x} G$ be the crossed product of a von Neumann algebra and a locally, compact abelian group $G$.

(a) The formula

$$
T x=\int_{\hat{G}} \hat{\alpha}_{p}(x) d p, \quad x \in\left(M \otimes_{x} G\right)_{+}
$$

defines a n.f.s. operator valued weight from $M \otimes_{x} G$ to $\pi(M)$.

(b) $T$ satisfies

$$
\begin{array}{ll}
T\left(\mu\left(x^{\sharp} * x\right)\right)=\pi\left(\left(x^{\sharp} * x\right)(e)\right), & x \in K(G, M) \\
T\left(\lambda(s) x \lambda(s)^{*}\right)=\lambda(s) T(x) \hat{\lambda}(s)^{*}, & x \in\left(M \otimes_{\alpha} G\right)_{+}, s \in G .
\end{array}
$$

(c) For any $\varphi \in P(M)$, the dual weight $\tilde{\varphi}$ on $M \otimes_{x} G$ is given by the formula $\tilde{\varphi}$ $=\left(\varphi \circ \pi^{-1}\right) \circ T$.

LeMmA 1.2. Let $f \in K(G)$, and assume that $\hat{f}(p)=\int_{G} \overline{\langle p, s\rangle} f(s) d s \geqq 0$ for any $p \in \hat{G}$. Then

$$
\int_{\hat{G}} \hat{f}(p) d p=f(e)
$$

Proof. By Bochner's theorem [10, p. 19] it follows that

$$
\int_{G} f(s) \varphi(s) d s \geqq 0 \quad \text { for any } \varphi \in P(G) .
$$


In particular

$$
\int_{G} f(s)\left(g^{*} * g\right)(s) d s \geqq 0, \quad g \in K(G) .
$$

Hence $f$ is positive definite. Thus by $\left[10\right.$, p. 22] it follows that $\hat{f} \in L^{1}(\hat{G})$, and that

$$
f(e)=\int_{\hat{G}} \hat{f}(p) d p .
$$

Lemma 1.3. Let $\varphi$ be a n.f.s. weight on a von Neumann algebra $R$, and put

$$
\psi(x)=c \varphi\left(u x u^{*}\right), \quad x \in \mathbf{R}_{+}
$$

where $u$ is a unitary operator in $R$ and $c>0$.

Then

$$
(D \psi: D \varphi)_{t}=c^{i t} u^{*} \sigma_{t}^{\varphi}(u)
$$

Proof. Put $\omega=\varphi\left(u \cdot u^{*}\right)$. Then by [3, lemma 1.2.3] we get

$$
(D \psi: D \varphi)_{t}=(D \psi: D \omega)_{t}(D \omega: D \varphi)_{t}=c^{i t} u^{*} \sigma_{t}^{\varphi}(u) .
$$

Proof of Theorem 1.1. (a) Put

$$
\langle\varphi, T x\rangle=\int_{\hat{G}}\left\langle\varphi, \hat{\alpha}_{p}(x)\right\rangle d p, \quad x \in\left(M \otimes_{\alpha} G\right)_{+}, \varphi \in\left(M \otimes_{\alpha} G\right)_{*}^{+} .
$$

Then $T x$ belongs to the extended positive part of $M \otimes_{\alpha} G$. Moreover $T x$ is invariant under the extension of $\hat{\alpha}_{p}$ to $\left(M \otimes_{\alpha} G\right)_{+}$. Hence as in the proof of [7, lemma 5.2] we conclude that $T x$ belongs to the extended positive part of the fixpoint algebra for $\hat{\alpha}$, i.e., $T x \in \pi(M)_{+}$. It is easy to check that $T$ is a normal, faithful operator valued weight from $M \otimes_{\alpha} G$ to $\pi(M)$. The semifiniteness of $T$ will follow, when (b) is proved.

(b) Let $x \in K(G, M)$, and let $\omega$ be a positive, normal functional on $M \otimes_{\alpha} G$. Then

$$
0 \leqq\left\langle\omega, \hat{\alpha}_{p}\left(\mu\left(x^{\#} * x\right)\right)\right\rangle=\int_{G} \overline{\langle p, s\rangle}\left\langle\omega, \lambda(s) \pi\left(\left(x^{\#} * x\right)(s)\right)\right\rangle d s .
$$

Thus the function

$$
s \rightarrow\left\langle\omega, \lambda(s) \pi\left(\left(x^{\#} * x\right)(s)\right)\right\rangle
$$

satisfies the conditions of lemma 1.2. Hence 


$$
\left\langle\omega, T\left(\mu\left(x^{\sharp} * x\right)\right)\right\rangle=\int_{\hat{G}}\left\langle\omega, \hat{\alpha}_{p}\left(\mu\left(x^{\sharp} * x\right)\right)\right\rangle d p=\left\langle\omega, \pi\left(\left(x^{\sharp} * x\right)(e)\right)\right\rangle
$$

or equivalently $T\left(\mu\left(x^{\#} * x\right)\right)=\pi\left(\left(x^{\#} * x\right)(e)\right)$. From this formula it follows that $\left\|T\left(\mu(x)^{*} \mu(x)\right)\right\|<\infty$ for any $x \in K(G, M)$. Hence $T$ is semifinite. The second formula in (b) follows easily, using that for $x \in M \otimes_{\alpha} G, s \in G$ and $p \in \hat{G}$ we have

$$
\alpha_{p}\left(\lambda(s) x \lambda(s)^{*}\right)=\lambda(s) \hat{\alpha}_{p}(x) \lambda(s)^{*} .
$$

(c) For $\varphi \in P(M)$ we put $\bar{\varphi}=\left(\varphi \circ \pi^{-1}\right) \circ T$. We will prove that $\bar{\varphi}=\tilde{\varphi}$ by the characterisation of the dual weight given in [8, lemma 3.3]. Clearly $\bar{\varphi}$ is a n.f.s. weight on $M \otimes_{\alpha} G$ and by [7, Theorem 4.7] we get

$$
\sigma_{t}^{\tilde{\varphi}}(\pi(x))=\sigma_{t}^{\varphi \circ \pi^{-1}}(\pi(x))=\pi\left(\sigma_{t}^{\varphi}(x)\right), \quad x \in M, \varphi \in P(M) .
$$

$\left({ }^{* *}\right) \quad(D \bar{\psi}: D \bar{\varphi})_{t}=\left(D \psi \circ \pi^{-1}: D \psi \circ \pi^{-1}\right)_{t}=\pi\left((D \psi: D \varphi)_{t}\right), \quad \varphi, \psi \in P(M)$.

By (b) it follows that

$$
\begin{aligned}
\overline{\varphi \circ \alpha_{s}}(x) & =\varphi \circ \alpha_{s} \circ \pi^{-1}(T x) \\
= & \varphi \circ \pi^{-1}\left(\lambda(s) T x \lambda(s)^{*}\right) \\
= & \left(\varphi \circ \pi^{-1}\right) T\left(\lambda(s) x \lambda(s)^{*}\right)=\bar{\varphi}\left(\lambda(s) x \lambda(s)^{*}\right) .
\end{aligned}
$$

Hence by lemma 1.3 and the above formulas $\left({ }^{*}\right)$ and $\left({ }^{* *}\right)$ we have:

$$
\pi\left(\left(D \varphi \circ \alpha_{s}: D \varphi\right)_{t}\right)=\left(D \overline{\varphi \circ \alpha_{s}}: D \bar{\varphi}\right)_{t}=\lambda(s)^{*} \sigma_{t}^{\bar{\varphi}}(\lambda(s))
$$

or equivalently

$(* * *) \quad \sigma_{t}^{\bar{\varphi}}(\lambda(s))=\lambda(s) \pi\left(\left(D \varphi \circ \alpha_{s}: D \varphi\right)_{t}\right)$.

By (b) we get for $x \in K(G, M)$

$$
\bar{\varphi}\left(\mu\left(x^{\#} * x\right)\right)=\left(\varphi \circ \pi^{-1}\right) \circ T\left(\mu\left(x^{\#} * x\right)\right)=\varphi\left(\left(x^{\#} * x\right)(e)\right) .
$$

Thus by $[8$, lemma 3.3$]$ it follows that $\bar{\varphi}=\tilde{\varphi}$.

\section{The canonical weight on $\mathscr{L}(G)$.}

Let $G$ be a locally compact group with a fixed Haar measure $d s$, and let $\Delta_{G}(s)$ be the module function on $G$. We let $l$ (respectively $r$ ) denote the left (respectively the right) regular representation of $G$ on $L^{2}(G)$.

$$
\begin{array}{ll}
(l(s) f)(t)=f\left(s^{-1} t\right), & f \in L^{2}(G) \\
(r(s) f)(t)=\Delta_{G}^{\frac{1}{G}}(s) f(t s), & f \in L^{2}(G) .
\end{array}
$$


Moreover $\mathscr{L}(G)$ and $\mathscr{R}(G)$ denote the von Neumann algebras generated by $l(G)$ and $r(G)$. In this situation $\mathscr{L}(G)^{\prime}=\mathscr{R}(G)$ (cf. [5, $\left.\S 13\right]$ ). As usual $K(G)$ denotes the set of continuous functions on $G$ with compact support.

Definition 2.1. (1) $f \in L^{2}(G)$ is left bounded if there exists a bounded operator $\pi_{l}(f)$ on $L^{2}(G)$ such that $\pi_{l}(f) g=f * g, \forall g \in K(G)$.

(2) $g \in L^{2}(G)$ is right bounded if there exists a bounded operator $\pi_{r}(g)$ on $L^{2}(G)$ such that $\pi_{r}(g) f=f * g, \forall f \in K(G)$.

For any complex function $f$ on $G$ we put

$$
f^{\sharp}(s)=\Delta_{G}(s)^{-1} \bar{f}\left(s^{-1}\right) \quad f^{*}(s)=\Delta_{G}(s)^{-\frac{1}{2}} \bar{f}\left(s^{-1}\right) \quad f^{b}(s)=\bar{f}\left(s^{-1}\right) .
$$

\#, * and ${ }^{b}$ are involutions of the convolution algebra $K(G)$. Moreover * is an isometry on $L^{2}(G)$.

The algebra $K(G)$ is a left Hilbert algebra with involution * and the usual inner product from $L^{2}(G)$. Note that our definition of * differs from $[5, \S 13]$. We have chosen ${ }^{\#}, *, b$, such that they correspond to the Tomita algebra structure of $K(G)$ (cf. $[12, \S 2]$ ). It is not hard to verify that the left (respectively right) bounded elements relative to the left Hilbert algebra $K(G)$ (cf. [2, definition 2.1]) coincide with the left (respectively right) bounded elements in the sense of the above definition 2.1 .

Definition 2.2. The canonical weight $\Omega$ on $\mathscr{L}(G)_{+}$is the n.f.s. weight associated with the left Hilbert algebra $K(G)$. Hence by [2. Definition 2.12]

$$
\Omega(x)=\left\{\begin{array}{cl}
\|f\|_{2}^{2} & \text { if } x=\pi_{l}(f)^{*} \pi_{l}(f), f \text { left bounded } \\
\infty & \text { otherwise }
\end{array}\right.
$$

The closure of the involution $f \rightarrow f^{\sharp}, f \in K(G)$, in the completion $L^{2}(G)$ of $K(G)$ has the polar decomposition $J \Delta^{\frac{1}{2}}$ where $\Delta$ is the densely defined multiplication operator on $L^{2}(G)$ given by

$$
(\Delta f)(s)=\Delta_{G}(s) f(s), \quad f \in L^{2}(G), \Delta_{G} f \in L^{2}(G),
$$

and $J f=f^{*}, f \in L^{2}(G)$. Hence the modular automorphism group $\sigma_{t}^{\Omega}$ associated with $\Omega$ is

$$
\sigma_{t}^{\Omega}(x)=\Delta^{i t} x \Delta^{-i t}, \quad x \in \mathscr{L}(G) .
$$

In particular

$$
\sigma_{t}^{\Omega}(l(s))=\Delta_{G}(s)^{i t} l(s), \quad s \in G .
$$

Note that $\Omega$ is a trace if and only if $G$ is unimodular. 
Lemma 2.3. Let $f, g \in L^{2}(G)$ such that $f$ is left bounded and $g$ is right bounded. Then

$$
\pi_{l}(f) \cdot g=\pi_{r}(g) \cdot f .
$$

Proof. Let $\left(u_{i}\right)_{i \in I}$ be an approximating unit for $G$ in the sense of $[5, \S 13]$. Then $\pi_{l}\left(u_{i}\right) \rightarrow 1$ strongly and $\pi_{r}\left(u_{i}\right) \rightarrow 1$ strongly. Hence

$$
\begin{aligned}
\pi_{l}(f) g & =\lim \pi_{l}(f) \pi_{l}\left(u_{i}\right) g=\lim \pi_{l}(f) \pi_{r}(g) u_{i} \\
& =\lim \pi_{r}(g) \pi_{l}(f) u_{i}=\lim \pi_{r}(g) \pi_{r}\left(u_{i}\right) f=\pi_{r}(g) f .
\end{aligned}
$$

Let $P(G)$ be the set of continuous positive definite functions on $G$. For $\varphi, \psi \in P(G)$ we write

$$
\varphi \ll \psi \quad \text { if } \quad \psi-\varphi \in P(G)
$$

and

$$
\varphi \ll \delta \quad \text { if } \quad \int_{G} \varphi(s)\left(f^{\sharp} * f\right)(s) d s \leqq\left(f^{\sharp} * f\right)(e) \forall f \in K(G)
$$

$(\delta=$ Dirac measure in $e)$.

Let $A(G)$ be the Fourier algebra on $G$ (cf. [6]). It is well known that the following conditions are equivalent:

(i) $\varphi \in P(G) \cap A(G)$

(ii) $\varphi=f * f^{b}, \quad f \in L^{2}(G)$

(iii) There exists a positive normal functional $\omega_{\varphi}$ on $\mathscr{L}(G)$ such that $\omega_{\varphi}(l(s))$ $=\varphi(s) \forall s \in G$.

Moreover $\varphi \rightarrow \omega_{\varphi}$ is a bijection of $P(G) \cap A(G)$ onto the positive part of the predual of $\mathscr{L}(G)$.

Proposition 2.4. Let $\varphi \in P(G)$. The following conditions are equivalent

(1) $\varphi \ll \delta$

(2) $\varphi \in A(G)$ and $\omega_{\varphi}(x) \leqq \Omega(x) \forall x \in \mathscr{L}(G)_{+}$.

Proof. (2) $\Rightarrow$ (1). Assume that $\varphi$ satisfies (2). Then $\forall f \in K(G)$ :

$$
\begin{aligned}
\int_{G} \varphi(s)\left(f^{\sharp} * f\right)(s) d s & =\omega_{\varphi}\left(l(f)^{*} l(f)\right) \\
& \leqq \Omega\left(l(f)^{*} l(f)\right)=\|f\|_{2}^{2}=\left(f^{\sharp} * f\right)(e) .
\end{aligned}
$$

(1) $\Rightarrow(2)$. The first part of the proof is analogue to the proof of $[1$, proposition 2.4]. Let $\varphi \ll \delta$ and let $\left(\pi_{\varphi}, H_{\varphi}, \xi_{\varphi}\right)$ be the cyclic representation of $G$ induced by $\varphi$ (cf. $[5, \S 13]$ ). For $f \in K(G)$ 


$$
\left\|\pi_{\varphi}(f) \xi_{\varphi}\right\|^{2}=\left\langle\varphi, f^{\sharp} * f\right\rangle \leqq\left(f^{\sharp} * f\right)(e)=\|f\|_{2}^{2} .
$$

Hence there is a bounded operator $T: L^{2}(G) \rightarrow H_{\varphi}$ such that $T f=\pi_{\varphi}(f) \xi_{\varphi}$, $\forall f \in K(G) .\|T\| \leqq 1$ and $T$ has dense range, because $\xi_{\varphi}$ is cyclic. A simple calculation shows that $T$ is a coupling operator for $\pi_{\varphi}$ and the left regular representation $l$ :

$$
T l(f)=\pi_{\varphi}(f) T, \quad \forall f \in K(G) .
$$

Let $T=U|T|$ be the polar decomposition of $T$. Then $U U^{*}=1$ and $U /(f)$ $=\pi_{\varphi}(f) U^{*}$. Therefore $\pi_{\varphi}$ is equivalent to a subrepresentation of the left regular representation. If we put $\xi=U^{*} \xi_{\varphi} \in L^{2}(G)$ and $g=\bar{\xi}$ (complex conjugate) then for $s \in G$

$$
\begin{aligned}
\varphi(s)=\left(\pi_{\varphi}(s) \xi_{\varphi} \mid \xi_{\varphi}\right) & =(l(s) \xi \mid \xi) \\
& =\int_{G} \xi\left(s^{-1} t\right) \bar{\xi}(t) d t=\left(g * g^{\jmath}\right)(s) .
\end{aligned}
$$

Hence $\varphi=g * g^{\prime}$, which proves that $\varphi \in A(G)$. Moreover

$$
\omega_{\varphi}(x)=(x \xi \mid \xi), \quad \forall \dot{x} \in \mathscr{L}(G) .
$$

For $f \in K(G)$

$$
f * \xi=\pi_{l}(f) \xi=U^{*} \pi_{\varphi}(f) \xi_{\varphi}=U^{*} T f=|T| f .
$$

Hence $\xi$ is right bounded, and $\pi_{r}(\xi)=|T|$. In particular $\left\|\pi_{r}(\xi)\right\| \leqq 1$. For $f \in L^{2}(G), f$ left bounded we get by lemma 2.3 that

$$
\begin{aligned}
\omega_{\varphi}\left(\pi_{l}(f)^{*} \pi_{l}(f)\right)=\left\|\pi_{l}(f) \xi\right\|_{2}^{2} & =\left\|\pi_{r}(\xi) f\right\|_{2}^{2} \\
& \leqq\|f\|_{2}^{2}=\Omega\left(\pi_{l}(f)^{*} \pi_{l}(f)\right) .
\end{aligned}
$$

Hence $\omega_{\varphi}(x) \leqq \Omega(x), \forall x \in \mathscr{L}(G)_{+}$.

Corollary 2.5 .

(1) $\Omega(x)=\sup _{\varphi \ll \delta} \omega_{\varphi}(x), \quad x \in \mathscr{L}(G)_{+}$.

(2) The set

$$
\mathscr{F}=\{\varphi \in P(G) \mid \exists \varepsilon>0: \varphi \ll(1-\varepsilon) \delta\}
$$

is directed with respect to the ordering $\ll$, (i.e. for any $\varphi_{1}, \varphi_{2} \in \mathscr{F}$ there exists $\varphi \in \mathscr{F}$ which dominates $\varphi_{1}$ and $\varphi_{2}$ ).

Proof. (1). By propositoon 2.4 the map $\varphi \rightarrow \omega_{\varphi}$ is a bijection of $\{\varphi \in P(G) \mid \varphi \ll \delta\}$ onto $\left\{\omega \in \mathscr{L}(G)_{*}^{+} \mid \omega \leqq \Omega\right\}$. Since $\Omega$ is normal

$$
\Omega(x)=\sup _{\omega \leqq \Omega} \omega(x), \quad x \in \mathscr{L}(G)_{+} .
$$


Hence (1).

(2). The map $\varphi \rightarrow \omega_{\varphi}$ is a bijective order isomorphism of $\{\varphi \in P(G) \mid \exists \varepsilon>0: \varphi \ll(1-\varepsilon) \delta\}$ onto $\left\{\omega \in L(G)_{*}^{+} \mid \exists \varepsilon>0: \omega \leqq(1-\varepsilon) \Omega\right\}$. According to [13, Theorem 13.8] the latter is directed. Hence (2) is proved.

Corollary 2.6. For $x \in \mathscr{L}(G)_{+}$and $s \in G$

$$
\Omega\left(l(s) x l(s)^{*}\right)=\Delta_{G}(s) \Omega(x) .
$$

Proof. Since for $s, t \in G$ and $\varphi \in P(G) \cap A(G)$ we have

$$
\omega_{\varphi}\left(l(s) l(t) l(s)^{*}\right)=\varphi\left(s t s^{-1}\right)=\omega_{\varphi\left(s \cdot s^{-1}\right)}(l(t))
$$

it follows that

$$
\omega_{\varphi}\left(\lambda(s) x \lambda(s)^{*}\right)=\omega_{\varphi\left(s \cdot s^{-1}\right)}(x), \quad x \in \mathscr{L}(G), s \in G .
$$

An easy computation shows that

$$
\varphi \ll \delta \Leftrightarrow \varphi\left(s \cdot s^{-1}\right) \ll \Delta_{G}(s) \delta .
$$

Hence by corollary $2.5(1)$ we get

$$
\Omega\left(\lambda(s) x \lambda(s)^{*}\right)=\Delta_{G}(s) \Omega(x), \quad x \in \mathscr{L}(G)_{+}, s \in G .
$$

Corollary 2.7. For $f \in L^{1}(G)$

$$
\sup _{\varphi \ll \delta} \int_{G} \varphi(s)\left(f^{\#} * f\right)(s) d s=\int_{G}|f(s)|^{2} d s .
$$

(The integral on the right side may be infinite).

Proof. By corollary 2.5 the above formula is equivalent to

$$
\Omega\left(l(f)^{*} l(f)\right)=\int_{G}|f(s)|^{2} d s, \quad f \in L^{1}(G) .
$$

If $f \in L^{1}(G) \cap L^{2}(G)$ the above formula is trivial, since in this case $f$ is left bounded and $l(f)=\pi_{l}(f)$. Let now $f \in L^{1}(G) \backslash L^{2}(G)$. We shall prove that $\Omega\left(l(f)^{*} l(f)\right)=\infty$. Assume that $\Omega\left(l(f)^{*} l(f)\right)<\infty$, then $l(f)=\pi_{l}(g)$ for some left bounded function $g \in L^{2}(G)$.

Hence for any $h \in K(G)$ we have $f * h=g * h$. However, this implies that $g=h$ a.e., which contradicts that $g \dot{\notin} L^{2}(G)$. This completes the proof.

Lemma 2.8. If $f, g \in L^{2}(G)$ are left bounded, then $g^{\#} * f$ is a continuous, left bounded $L^{2}$-function, and 


$$
\pi_{l}\left(g^{\#} * f\right)=\pi_{l}(g)^{*} \pi_{l}(f) .
$$

Proof. Since $f, g \in L^{2}(G), g^{\#} * f$ is well defined and continuous. The formula $\pi_{l}\left(g^{\sharp} * f\right)=\pi_{l}(g)^{*} \pi_{l}(f)$ is trivial if $f, g \in K(G)$. In the general case we can choose sequences $\left(f_{n}\right)_{n \in N}$ and $\left(g_{n}\right)_{n \in N}$ in $K(G)$ such that $\left\|f_{n}-f\right\|_{2} \rightarrow 0$ and $\left\|g_{n}-g\right\|_{2}$ $\rightarrow 0$. Since $\left\{\pi_{l}(f) \mid f\right.$ left bounded $\}$ is a left ideal in $\mathscr{L}(G)$, there exists a left bounded $L^{2}$-function $a$ such that $\pi_{l}(a)=\pi_{l}(g)^{*} \pi_{l}(f)$. Hence for $h, k \in K(G)$ :

$$
\begin{aligned}
\lim _{n \rightarrow \infty}\left(g^{\sharp} * f_{n} * h \mid k\right) & =\lim _{n \rightarrow \infty}\left(f_{n} * h \mid g_{n} * k\right) \\
& =(f * h \mid g * k) \\
& =\left(\pi_{l}(g)^{*} \pi_{l}(f) h \mid k\right)=(a * h \mid k) .
\end{aligned}
$$

However $g_{n}^{\#} * f_{n} \rightarrow g^{\#} * f$ uniformly on compact sets. Hence

$$
\int_{G}\left(\left(g^{\#} * f\right) * h\right)(s) \bar{k}(s) d s=(a * h \mid k)=\int_{G}(a * h)(s) \bar{k}(s) d s .
$$

This implies that

$$
\left(g^{\sharp} * f\right) * h=a * h \text { a.e. for any } h \in K(G) .
$$

Hence, $g^{\#} * f=a$ a.e. Thus $g^{\sharp} * f$ is a left bounded $L^{2}$-function and

$$
\pi_{l}\left(g^{\sharp} * f\right)=\pi_{l}(a)=\pi_{l}(g)^{*} \pi_{l}(f) .
$$

As usual we put

$$
n_{\Omega}=\left\{x \in \mathscr{L}(G) \mid \Omega\left(x^{*} x\right)<\infty\right\}
$$

and

$$
m_{\Omega}=n_{\Omega}^{*} n_{\Omega}=\operatorname{span}\left\{z^{*} y \mid y, z \in n_{\Omega}\right\}
$$

Proposition 2.9.

1) $n_{\Omega}=\left\{\pi_{l}(f) \mid f\right.$ left bounded $\}$

2) $m_{\Omega}^{+}=\left\{\pi_{l}(f) \mid f\right.$ continuous, left bounded and $\left.\pi_{l}(f) \geqq 0\right\}$.

3) If $\pi_{l}(f) \in m_{\Omega}^{+}$, then $f$ is almost everywhere equal to a unique continuous function $f_{1}$ and $\Omega\left(\pi_{l}(f)\right)=f_{1}(e)$.

Proof. (1) follows from the definition of $\Omega$. (2). Let $x \in m_{\Omega}^{+}$, then $x$ $=\pi_{l}(f)^{*} \pi_{l}(f)$ for some left bounded $L^{2}$-function $f$. By lemma $2.8, g=f^{\#} * f$ is a continuous left bounded $L^{2}$-function, and $x=\pi_{l}(g) \geqq 0$.

Conversely let $f$ be a continuous left bounded $L^{2}$-function such that $\pi_{l}(f) \geqq 0$. Since the isometric involution $J: f \rightarrow f^{*}$ in $L^{2}(G)$ maps the set of left bounded elements onto the set of right bounded elements and since $\pi_{r}\left(f^{*}\right)=J \pi_{l}(f) J$ (cf. 
[2]), it follows that for some $K>0$, we have $0 \leqq \pi_{r}\left(f^{*}\right) \leqq K$. Since for any $g \in K(G)$

$$
\int_{G} f^{*}(s)\left(g^{\sharp} * g\right)(s) d s=\left(f^{*} \mid \pi_{l}(\bar{g})^{*} \bar{g}\right)=\left(\bar{g} * f^{*} \mid \bar{g}\right)=\left(\pi_{r}\left(f^{*}\right) \bar{g} \mid \bar{g}\right)
$$

we get

$$
0 \leqq \int_{G} f^{*}(s)\left(g^{\sharp} * g\right)(s) d s \leqq K\|g\|_{2}^{2}=K\left(g^{*} * g\right)(e) .
$$

This proves that $f^{*} \in P(G)$ and that $f^{*} \ll K \cdot \delta$.

Hence by the proof of proposition 2.4 we get

$$
f^{*}=h * h^{b}
$$

for some right bounded $L^{2}$-function $H$. Thus

$$
f=\left(f^{*}\right)^{*}=\left(h^{b}\right)^{*} * h^{*}=k^{\sharp} * k
$$

where $k=h^{*}$ is left bounded. Hence by lemma 2.8

$$
\pi_{l}(f)=\pi_{l}(k)^{*} \pi_{l}(k)
$$

which proves that $\pi_{l}(f) \in \dot{m}_{\Omega}^{+}$.

(3) Assume that $\pi_{l}(f) \in m_{\Omega}^{+}$. Then $\pi_{l}(f)=\pi_{l}(g) * \pi_{l}(g)$ for a left bounded $L^{2}$ function $g$. By lemma 2.8 we get $f * h=\left(g^{\#} * g\right) h$ for any $h \in K(G)$. Hence $f$ $=g^{\#} * g$ a.e. However, $g^{\#} * g$ is continuous and

$$
\Omega\left(\pi_{l}(f)\right)=\Omega\left(\pi_{l}(g)^{*} \pi_{l}(g)\right)=\|g\|_{2}^{2}=\left(g^{\#} * g\right)(e) .
$$

Since the support of the Haar measure is the whole group $G$, it follows that if $f$ $=f_{1}$ a.e. and $f_{1}$ is continuous, then $f_{1}=g^{\#} * g$.

Corollary 2.10. If $f \in K(G)$ and $\int_{G} f(s) \varphi(s) d s \geqq 0$ for any $\varphi \in P(G)$ then

$$
\sup _{\varphi \ll \delta} \int_{G} f(s) \varphi(s) d s=f(e) \text {. }
$$

Proof. Since $f \in K(G), f$ is left bounded and

$$
\pi_{l}(f)=l(f)=\int_{G} f(s) l(s) d s .
$$

Hence

$$
\omega_{\varphi}\left(\pi_{l}(f)\right)=\int_{G} f(s) \varphi(s) d s \geqq 0 \quad \text { for any } \varphi \in P(G) \cap A(G)
$$


which proves that $\pi_{l}(f) \geqq 0$. Hence by proposition 2.9 we have

$$
\pi_{l}(f) \in m_{\Omega}^{+} \quad \text { and } \quad \Omega\left(\pi_{l}(f)\right)=f(e) .
$$

By corollary 2.5

$$
\sup _{\varphi \ll \delta} \int_{G} f(s) \varphi(s) d s=\Omega\left(\pi_{l}(f)\right)=f(e) .
$$

\section{Crossed products with arbitrary groups.}

THEOREM 3.1. Let $M \otimes_{\alpha} G$ be the crossed product of a von Neumann algebra $M$ with a locally compact group $G$.

(a) For each continuous, positive definite function $\varphi$ on $G$ there is a unique $\sigma$ weakly continuous linear map $E_{\varphi}$ on $M \otimes_{\alpha} G$, such that

$$
E_{\varphi}(a x b)=a E_{\varphi}(x) b, \quad x \in M \otimes_{\alpha} G, a, b \in \pi(M),
$$

and

$$
E_{\varphi}(\lambda(s))=\varphi(s) \hat{\lambda}(s), . \quad s \in G .
$$

Each $E_{\varphi}$ is completely positive, and $E_{\varphi+\psi}=E_{\varphi}+E_{\psi}$ for $\varphi, \psi \in P(G)$.

(b) The formula

$$
T x=\sup _{\varphi \ll \delta} E_{\varphi} x, \quad x \in\left(M \otimes_{\alpha} G\right)_{+}
$$

defines a n.f.s. operator valued weight $T$ from $M \otimes_{\alpha} G$ to $\pi(M)$.

(c) $T$ satisfies

$$
\begin{array}{ll}
T\left(\mu\left(x^{\#} * x\right)\right)=\pi\left(\left(x^{*} * x\right)(e)\right), & x \in K(G, M) . \\
T\left(\lambda(s) x \lambda(s)^{*}\right)=\Delta_{G}(s) \lambda(s) T(x) \lambda(s)^{*}, & x \in\left(M \otimes_{\alpha} G\right)_{+}, s \in G .
\end{array}
$$

(d) For any $\varphi \in P(M)$ the dual weight $\tilde{\varphi}$ on $M \otimes_{\alpha} G$ is given by $\tilde{\varphi}=$ $\left(\varphi \circ \pi^{-1}\right) \circ T$.

Lemma 3.2. Let $\varphi \in P(G)$. There exists a family $\left(a_{i}\right)_{i \in I}$ of bounded, continuous functions on $G$, such that

$$
\varphi\left(s t^{-1}\right)=\sum_{i \in I} a_{i}(s) \overline{a_{i}}(t), \quad s, t \in G
$$

where the series converges absolutely, and uniformly on compact subsets of $G \times G$.

Proof. Let $\left(\pi_{\varphi}, H_{\varphi}, \xi_{\varphi}\right)$ be the representation of $G$ induced by $\varphi$ (cf. $[5, \S 13]$ ), and let $\left(e_{i}\right)_{i \in I}$ be a basis for $H_{\varphi}$. Put $a_{i}(s)=\left(\pi_{\varphi}(s) e_{i} \mid \xi_{\varphi}\right), i \in I$, then 


$$
\begin{aligned}
\varphi\left(s t^{-1}\right) & =\left(\pi_{\varphi}\left(t^{-1}\right) \xi_{\varphi} \mid \pi_{\varphi}\left(s^{-1}\right) \xi_{\varphi}\right) \\
& =\sum_{i \in I}\left(\pi_{\varphi}\left(t^{-1}\right) \xi_{\varphi} \mid e_{i}\right)\left(e_{i} \mid \pi_{\varphi}\left(s^{-1}\right) \xi_{\varphi}\right) \\
& =\sum_{i \in I} a_{i}(s) \overline{a_{i}}(t)
\end{aligned}
$$

where the series converges absolutely for any $s, t \in G$. For $s=t$ we get $\sum_{i \in I}\left|a_{i}(s)\right|^{2}=\varphi(e)$. Hence by Dini's theorem this series converges uniformly om compact subsets of $G$. By Cauchy-Schwartz inequality it follows that for any finite subset $J$ of $I$ we have:

$$
\begin{aligned}
\left|\varphi\left(s t^{-1}\right)-\sum_{i \in J} a_{i}(s) \overline{a_{i}}(t)\right|^{2} & =\left|\sum_{i \in I J J} a_{i}(s) \overline{a_{i}}(t)\right|^{2} \\
& \leqq\left(\sum_{i \in I J J}\left|a_{i}(s)\right|^{2}\right)\left(\sum_{i \in I \backslash J}\left|a_{i}(t)\right|^{2}\right) .
\end{aligned}
$$

Therefore $\sum_{i \in I} a_{i}(s) \overline{a_{i}}(t)$ converges uniformly to $\varphi\left(s t^{-1}\right)$ on compact subsets of $G \times G$.

Lemma 3.3. For any $g \in L^{1}(G)$

$$
\sup _{\varphi \ll \delta} \iint_{G \times G} \varphi\left(s t^{-1}\right) g(s) \bar{g}(t) d s d t=\int_{G}|g(s)|^{2} \Delta_{G}(s) d s .
$$

Proof. It is easily seen that

$$
\iint_{G \times G} \varphi\left(s t^{-1}\right) g(s) \bar{g}(t) d s d t=\int_{G} \varphi(s)\left(g * g^{\sharp}\right)(s) d s .
$$

Put $f=g^{\#}$. Then

$$
\int_{G}|g(s)|^{2} \Delta_{G}(s) d s=\int_{G}|f(s)|^{2} d s .
$$

Hence the lemma follows from corollary 2.7.

It is no loss of generality to assume that the von Neumann algebra $M$ in Theorem 3.1 acts on a Hilbert space $H$, such that there exists a strongly continuous unitary representation $s \rightarrow u(s)$ of $G$ on $H$, satisfying

$$
\alpha_{s} x=u(s) x u(s)^{*}, \quad x \in M, s \in G .
$$

(see for instance $[8, \S 1.2])$. We let $(\pi, \lambda)$ be the covariant representation of $(M, G, \alpha)$ on $L^{2}(G, H)$ that generates the crossed product. 
LEMMA 3.4. Under the above assumptions

$$
\pi(M)=\left(M \otimes_{\alpha} G\right) \cap\left(B(H) \otimes L^{\infty}(G)\right) .
$$

Proof. Let $U$ be the unitary operator on $L^{2}(G, H)$ defined by

$$
(U \xi)(s)=u(s) \xi(s), \quad \xi \in L^{2}(G, H) .
$$

Then, as in the proof of $[8$, lemma 3.6] we get

$$
\begin{aligned}
\pi(M) & =\left(M^{\prime} \otimes 1\right)^{\prime} \cap\left(U^{*}(1 \otimes \mathscr{R}(G)) U\right)^{\prime} \cap\left(1 \otimes L^{\infty}(G)\right)^{\prime} \\
& =\left(M \otimes_{\alpha} G\right) \cap\left(B(H) \otimes L^{\infty}(G)\right) .
\end{aligned}
$$

Lemma 3.5. (1) For each $\varphi \in P(G)$ there exsists a unique $\sigma$-weakly continuous map $F_{\varphi}$ on $B\left(L^{2}(G, H)\right)$, such that

$$
\begin{aligned}
& F_{\varphi}(a x b)=a F_{\varphi}(x) b, \quad x \in B\left(L^{2}(G, H)\right), a, b \in B(H) \otimes L^{\infty}(G) \\
& F_{\varphi}(\lambda(s))=\varphi(s) \lambda(s), \quad s \in G . \\
& F_{\varphi} \text { is completely positive, and } F_{\varphi+\psi}=F_{\varphi}+F_{\psi} \text { for } \varphi, \psi \in P(G) .
\end{aligned}
$$

(2) The formula

$$
S x=\sup _{\varphi \ll \delta} F_{\varphi}(x), \quad x \in\left(B\left(L^{2}(G, H)\right)\right)_{+}
$$

defines a normal, faithful operator valued weight from $B\left(L^{2}(G, H)\right)$ to $B(H) \otimes L^{\infty}(G)$.

Proof. (1). For convenience we put $K=L^{2}(G, H)$. Let $\varphi \in P(G)$. We have

$$
\varphi\left(s t^{-1}\right)=\sum_{i \in I} a_{i}(s) \overline{a_{i}}(t)
$$

where $\left(a_{i}\right)_{i \in I}$ is chosen as in lemma 3.2. In particular

$$
\varphi(e)=\sum_{i \in I}\left|a_{i}(s)\right|^{2}, \quad s \in G,
$$

where the series converges uniformly on compact sets, and therefore also in the $\sigma\left(L^{\infty}(G), L^{1}(G)\right)$ topology. We will not separate between $a_{i} \in L^{\infty}(G)$ and the corresponding multiplication operator on $L^{2}(G)$.

Thus

$$
\sum_{i \in I} a_{i} a_{i}^{*}=\varphi(e) 1 \quad(\sigma \text {-weakly })
$$

Put

$$
F_{\varphi}(x)=\sum_{i \in I}\left(1 \otimes a_{i}\right) x\left(1 \otimes a_{i}^{*}\right), \quad x \in B(K)
$$


Clearly $F_{\varphi}$ is a strictly positive, normal map on $B(K)$, and $F_{\varphi}$ satisfies

$$
F_{\varphi}(a x b)=a F_{\varphi}(x) b, \quad x \in B(K), a, b \in B(H) \otimes L^{\infty}(G) .
$$

For $\xi, \eta \in K$ we get

$$
\begin{aligned}
\left(F_{\varphi} \lambda(s) \xi \mid \eta\right) & =\sum_{i \in I}\left(\left(\lambda(s) 1 \otimes a_{i}^{*}\right) \dot{\xi} \mid\left(1 \otimes a_{i}^{*}\right) \eta\right) \\
& =\sum_{i \in I} \int_{G} \overline{a_{i}}\left(s^{-1} t\right) a_{i}(t)\left(\xi\left(s^{-1} t\right) \mid \eta(t)\right) d t \\
& =\int_{G}\left(\sum_{i \in I} \overline{a_{i}}\left(s^{-1} \bar{t}\right) a_{i}(t)\right)\left(\xi\left(s^{-1} t\right) \mid \eta(t)\right) d t \\
& =\varphi(s)(\lambda(s) \xi \mid \eta) .
\end{aligned}
$$

The above permutation of sum and integration is permitted also if $I$ is uncountable, because the series $\sum_{i \in I} a_{i}(s) \overline{a_{i}}(t)$ converges uniformly on compact sets of $G \times G$. Thus

$$
F_{\varphi}(\lambda(s))=\varphi(s) \lambda(s), \quad s \in G .
$$

Let $i$ be the identity on $B(H)$ and let $\gamma(s)$ be the left translations with $s \in G$ on $L^{\infty}(G)$, then for any $a \in B(H) \otimes L^{\infty}(G)$ we have

$$
\lambda(s) a \lambda(s)^{*}=(i \otimes \gamma(s))(a) .
$$

Therefore the set

$$
\mathscr{A}=\operatorname{span}\left\{(1 \otimes l(s)) a \mid a \in B(H) \otimes L^{\infty}(G), s \in G\right\}
$$

is a *subalgebra of $B(K)$, and the commutant $\mathscr{A}^{\prime}$ is $C_{K}$, because

$$
\mathscr{R}(G) \cap L^{\infty}(G)=\mathrm{C}_{L^{2}(G)} .
$$

Hence $\mathscr{A}$ is $\sigma$-weakly dense in $B(K)$, and thus the uniqueness of $F_{\varphi}$ follows. Clearly $F_{\varphi+\psi}=F_{\varphi}+F_{\psi}$ by the uniqueness of $F_{\varphi+\psi}$.

(2) It follows from (1) that for $\varphi, \psi \in P(G)$ :

$$
\varphi \ll \psi \Rightarrow F_{\varphi} x \leqq F_{\psi} x, \quad \forall x \in B(K)_{+} .
$$

Put

$$
\mathscr{F}=\{\varphi \in P(G) \mid \exists \varepsilon>0: \varphi \ll(1-\varepsilon) \delta\} .
$$

By corollary 2.5 (2) it follows that $\left(F_{\varphi} x\right)_{\varphi \in \mathscr{F}}$ is an increasing net of positive operators. Hence for each $x \in B(K)_{+}$the formula

$$
\langle\omega, S x\rangle=\sup _{\varphi<\delta}\left\langle F_{\varphi} x, \omega\right\rangle=\sup _{\varphi \in \mathscr{F}}\left\langle F_{\varphi} x, \omega\right\rangle, \quad \omega \in B(K)_{*}^{+}
$$


defines an element $S x$ in the extended positive part of $B(K)$. Moreover the map $x \rightarrow S x$ is homogeneous, additive, normal, and

$$
S\left(a^{*} x a\right)=a^{*}(S x) a, \quad a \in B(H) \otimes L^{\infty}(G) .
$$

We shall prove that $S x$ belongs to the extended positive part of $B(H) \otimes L^{\infty}(G)$. For $\xi \in K$ we let $A(\xi)$ denote the positive operator of rank one given by

$$
A(\xi) \eta=(\eta \mid \xi) \xi, \quad \eta \in K .
$$

For $\xi, \eta \in K\left(=L^{2}(G, H)\right)$ we get

$$
\begin{aligned}
\left(F_{\varphi} A(\xi) \eta \mid \eta\right) & =\sum_{i \in I}\left(A(\xi)\left(1 \otimes a_{i}^{*}\right) \eta \mid\left(1 \otimes a_{i}^{*}\right) \eta\right) \\
& =\sum_{i \in I}\left|\left(\left(1 \otimes a_{i}^{*}\right) \eta \mid \xi\right)\right|^{2} \\
& =\sum_{i \in I}\left|\int_{G} a_{i}(s)(\xi(s) \mid \eta(s)) d s\right|^{2} \\
& =\sum_{i \in I} \iint_{G \times G} a_{i}(s) \bar{a}_{i}(t)(\xi(s) \mid \eta(s)) \overline{(\xi(t) \mid \eta(t))} d s d t \\
& =\iint_{G \times G}\left(\sum_{i \in I} a_{i}(s) \overline{a_{i}}(t)\right)(\xi(s) \mid \eta(s)) \overline{(\xi(t) \mid \eta(t))} d s d t \\
& =\iint_{G \times G} \varphi\left(s t^{-1}\right)(\xi(s) \mid \eta(s)) \overline{(\xi(t) \mid \eta(t))} d s d t
\end{aligned}
$$

Hence by lemma 3.3.

$$
\left\langle\omega_{\eta}, S(A(\xi))\right\rangle=\sup _{\varphi \ll \delta}\left(F_{\varphi} A(\xi) \eta \mid \eta\right)=\int_{G}|(\xi(s) \mid \eta(s))|^{2} \Delta_{G}(s) d s .
$$

For any unitary $u \in L^{\infty}(G)\left(|u(s)|=1\right.$ locally a.e.) and any $\eta \in L^{2}(G, H)$ we have

$$
\begin{aligned}
\left\langle\omega_{\eta},\left(1 \otimes u^{*}\right) S(A(\xi))(1 \otimes u)\right\rangle=\left\langle\omega_{(1 \otimes u) \eta}, S(A(\xi))\right\rangle & \\
= & \int_{G}|(\xi(s) \mid u(s) \eta(s))|^{2} \dot{\Delta_{G}}(s) d s \\
= & \int_{G}|(\xi(s) \mid \eta(s))|^{2} \Delta_{G}(s) d s=\left\langle\omega_{\eta}, S(A(\xi))\right\rangle .
\end{aligned}
$$

Hence $S(A(\xi))$ is affiliated with $\left(1 \otimes L^{\infty}(G)\right)^{\prime}=B(H) \otimes L^{\infty}(G)$ for any $\xi \in L^{2}(G, H)$ (cf. $\left.[7, \S 1]\right)$.

Since the set of positive, finite rank operators in $B(K)$ is the positive part of a $\sigma$-weakly dense ideal, any $x \in B(K)_{+}$has the form 


$$
x=\sum_{j \in J} A\left(\xi_{j}\right), \quad \xi_{j} \in K .
$$

Thus, since $S$ is normal, it follows that

$$
S x \in\left(B(H) \otimes L^{\infty}(G)\right)_{+} \quad \text { for any } x \in B(K)_{+} .
$$

This proves that $S$ is a normal operator valued weight from $B(K)$ to $B(H) \otimes L^{\infty}(G)$. For $\xi \in K, \xi \neq 0$, we have

$$
\left\langle\omega_{\xi}, S(A(\xi))\right\rangle=\int_{G}\|\xi(s)\|^{4} \Delta_{G}(s) d s>0 .
$$

Since any $x \in B(K)_{+}$has the form $\sum_{j \in J} A\left(\xi_{j}\right)$ we conclude that $S$ is faithful.

Proof of Theorem 3.1. (a) Let $F_{\varphi}, \varphi \in P(G)$ and $S$ be as in lemma 3.6, and let $E_{\varphi}, \varphi \in P(G)$, and $T$ be their restrictions to $M \otimes_{\alpha} G$ and $\left(M \otimes_{\alpha} G\right)_{+}$ respectively. Clearly $E_{\varphi}$ satisfies the conditions in (a). Since the operators of the form $\lambda(s) \pi(a), s \in G, a \in M$ span a $\sigma$-weakly dense subset of $M \otimes_{\alpha} G$ the uniqueness of $E_{\varphi}$ follows.

(b) For $x \in\left(M \otimes_{\alpha} G\right)_{+}$we get

$$
T x=\sup _{\varphi \ll \delta} E_{\varphi} x=S x .
$$

Hence $T$ is a positive, normal, homogeneous, additive map of $\left(M \otimes_{\alpha} G\right)_{+}$into $\left(M \otimes_{\alpha} G\right)_{+}$.

\section{Moreover}

$$
T\left(a^{*} x a\right)=a^{*} T(x) a, \quad x \in\left(M \otimes_{\alpha} G\right)_{+}, a \in \pi(M)=M \otimes 1 .
$$

By lemma 3.6 (b) it follows that $T x$ is affiliated with $B(H) \otimes L^{\infty}(G)$. Let

$$
T x=\int_{0}^{\infty} \lambda d e_{\lambda}+p \cdot \infty
$$

be the spectral resolution of $T x$ (cf. $[7, \S 1]$ ), then using lemma $3.4 e_{\lambda}$ and $p$ belong to

$$
\left(M \otimes_{\alpha} G\right) \cap\left(B(H) \otimes L^{\infty}(G)\right)=\pi(M) .
$$

Thus $T x \in \pi(M)_{+}$. Therefore $T$ is a normal operator valued weight from $M \otimes_{\alpha} G$ to $\pi(M)$. Clearly $T$ is faithful, since $S$ is faithful. The semifiniteness of $T$ will follow, when (c) is proved.

(c) For $x \in K(G, M)$ we put

$$
\mu(x)=\int_{G} \lambda(s) \pi(x(s)) d s
$$


as in section 1. By (a) we get for $\varphi \in P(G)$

$$
E_{\varphi}(\mu(x))=\int_{G} \varphi(s) \lambda(s) \pi(x(s)) d s .
$$

Hence for any $x \in K(G, M)$ and $\omega \in\left(M \otimes_{\alpha} G\right)_{*}^{+}$we have

$$
0 \leqq\left\langle\omega, E_{\varphi}\left(\mu\left(x^{\#} * x\right)\right)\right\rangle=\int_{G} \varphi(s)\left\langle\omega, \lambda(s) \pi\left(\left(x^{\#} * x\right)(s)\right)\right\rangle d s .
$$

Therefore the function

$$
s \rightarrow\left\langle\omega, \lambda(s) \pi\left(\left(x^{\#} * x\right)(s)\right)\right\rangle
$$

satisfies the conditions of corollary 2.10 .

Hence

$$
\left\langle\omega, T\left(\mu\left(x^{\#} * x\right)\right)\right\rangle=\sup _{\varphi \ll \delta}\left\langle\omega, E_{\varphi}\left(\mu\left(x^{\#} * x\right)\right)\right\rangle=\left\langle\omega, \pi\left(\left(x^{\#} * x\right)(e)\right)\right\rangle
$$

or equivalently $T\left(\mu\left(x^{\#} * x\right)\right)=\pi\left(\left(x^{\#} * x\right)(e)\right)$.

Since $\mu(K(G, M))$ is $\sigma$-weakly dense in $M \otimes_{\alpha} G$ it follows that $T$ is semifinite. To prove the second equation in (c) we will first verify the formula

$$
E_{\varphi}\left(\lambda(s) x \dot{\lambda}(s)^{*}\right)=\lambda(s) E_{\varphi\left(s \cdot s^{-1}\right)}(x) \lambda(s)^{*}
$$

for $x \in M \otimes_{\alpha} G$ and $s \in G$. It is enough to consider elements $x$ of the form $x$ $=\lambda(t) \pi(a), t \in G, a \in M$ :

$$
\begin{aligned}
E_{\varphi}\left(\lambda(s) \lambda(t) \pi(a) \lambda(s)^{*}\right) & =E_{\varphi}\left(\lambda\left(s t s^{-1}\right) \pi\left(\alpha_{s} a\right)\right) \\
& =\varphi\left(s t s^{-1}\right) \lambda\left(s t s^{-1}\right) \pi\left(\alpha_{s} a\right) \\
& =\varphi\left(s t s^{-1}\right) \lambda(s) \lambda(t) \pi(a) \lambda(s)^{*} \\
& =\lambda(s) E_{\varphi\left(s \cdot s^{-1}\right)}(\lambda(t) \pi(a)) \lambda(s)^{*}
\end{aligned}
$$

Hence $\left({ }^{*}\right)$ follows. Using that

$$
\varphi \ll \delta \Leftrightarrow \varphi\left(s \cdot s^{-1}\right) \ll \Delta_{G}(s) \delta
$$

we get as in the proof of corollary 2.6 that

$$
T\left(\lambda(s) x \lambda(s)^{*}\right)=\Delta_{G}(s) \lambda(s) T(x) \lambda(s)^{*}, \quad x \in\left(M \otimes_{\alpha} G\right)_{+}, s \in G .
$$

Thus (c) is proved.

(d) follows from (c) as in the abelian case, except for changes, due to the modular function $\Delta_{G}(s)$. Put $\bar{\varphi}=\left(\varphi \circ \pi^{-1}\right) \circ T, \varphi \in P(G)$ we get

$$
\overline{\varphi \circ \alpha_{s}}(x)=\Delta_{G}(s)^{-1} \bar{\varphi}\left(\lambda(s) x \lambda(s)^{*}\right), \quad x \in\left(M \otimes_{\alpha} G\right)_{+}, s \in G,
$$

and thus formula $\left({ }^{* * *}\right)$ in the proof of Theorem 1.1 must be changed to 


$$
\sigma_{t}^{\bar{\varphi}}(\lambda(s))=\Delta_{G}(s)^{i t} \lambda(s) \pi\left(\left(D \varphi \circ \alpha_{s}: D \varphi\right)_{t}\right) .
$$

This follows from lemma 1.3 (with $c=\Delta_{G}(s)^{-1}$ ). However, the conclusion $\bar{\varphi}=\tilde{\varphi}$ follows as in the abelian case.

Remark. Let $M \otimes_{\alpha} G$ be a crossed product with an abelian locally compact group $G$. Since there is only one operator valued weight $T$ from $M \otimes_{\alpha} G$ to $\pi(M)$ such that $\tilde{\varphi}=\left(\varphi \circ \pi^{-1}\right) \circ T$ for any $\varphi \in P(M)$ (cf. [7, corollary 5.4]) we get by Theorem 1.1 and Theorem 3.1 that

$$
\sup _{\varphi \ll \delta} E_{\varphi}(x)=\int_{\hat{G}} \hat{\alpha}_{p}(x) d p, \quad x \in\left(M \otimes_{\alpha} G\right)_{+} .
$$

This can also be proved directly: Let $\varphi \in P(G)$. By Bochner's theorem there is a unique Radon measure $v_{\varphi}$ on $\hat{G}$, such that

$$
\varphi(s)=\int_{\hat{G}} \overline{\langle p, s\rangle} d v_{\varphi}(p) .
$$

It is easy to check that

$$
E_{\varphi}(x)=\int_{\hat{G}} \overline{\langle p, s\rangle} \hat{\alpha}_{p}(x) d v_{\varphi}(p)
$$

satisfies the conditions of Theorem 3.1 (a). Moreover it is not hard to prove that

$$
\varphi \ll \delta \Leftrightarrow v_{\varphi} \leqq d p
$$

Hence

$$
\sup _{\varphi \ll \delta} E_{\varphi}(x)=\sup _{v \leqq d p} \int_{\hat{G}} \hat{\alpha}_{p}(x) d v(p)=\int_{\hat{G}} \hat{\alpha}_{p}(x) d p .
$$

Corollary 3.6. The dualisation map $\varphi \rightarrow \tilde{\varphi}$ has a natural extension to all normal weights on $M$, given by the formula

$$
\tilde{\varphi}=\left(\varphi \circ \pi^{-1}\right) \circ T .
$$

Moreover

(1) $\quad(\lambda \varphi)^{\tilde{}}=\lambda \tilde{\varphi}, \quad \lambda \geqq 0$, and $(\varphi+\psi)^{\tilde{\varphi}}=\tilde{\varphi}+\tilde{\psi}$.

(2) For $x \in K(G, M)$

$$
\tilde{\varphi}\left(\mu\left(x^{\#} * x\right)\right)=\varphi\left(\left(x^{\#} * x\right)(e)\right) .
$$

Proof. (1) is trivial. (2) follows from Theorem 3.1 (c). 
It is well known that when $M \otimes_{\alpha} G$ is the crossed product of a von Neumann algebra $M$ with a discrete group $G$ of automorphisms, then there exists a normal conditional expectation $\varepsilon$ from $M \otimes_{\alpha} G$ to $\pi(M)$, given by

$$
\varepsilon\left(\sum_{s \in G} \lambda(s) \pi(x(s))\right)=\pi(x(e))
$$

for any $M$-valued function $x$ on $G$, with finite support.

Corollary 3.7. Let $M \otimes_{\alpha} G$ be the crossed product of a von Neumann algebra by a discrete group of automorphisms. For any $\varphi \in P(M)$, the dual weight $\tilde{\varphi}$ on $M \otimes_{\alpha} G$ is given by

$$
\tilde{\varphi}=\varphi \circ \varepsilon
$$

where $\varepsilon$ is the above normal, conditional expectation from $M \otimes_{\alpha} G$ to $\pi(M)$.

Proof. Since $G$ is discrete, the set of positive, definite functions majorized by $\delta$ has a largest element, namely

$$
\dot{\varphi}_{0}(s)= \begin{cases}1 & s=e \\ 0 & s \neq e .\end{cases}
$$

Hence

$$
T x=\sup _{\varphi \ll \delta} E_{\varphi}(x)=E_{\varphi_{0}}(x), \quad x \in\left(M \otimes_{\alpha} G\right)_{+} .
$$

By Theorem 3.1 (a) it follows that $E_{\varphi_{0}}$ is a conditional expectation from $M \otimes_{\alpha} G$ to $\pi(M)$, and that

$$
E_{\varphi_{0}}(\lambda(s) \pi(a))=\left\{\begin{array}{cc}
\pi(a) & s=e \\
0 & s \neq e
\end{array}\right.
$$

for $s \in G$ and $a \in M$. This completes the proof.

REMARK. Let $T$ be the operator valued weight in Theorem 3.1, and assume that $G$ is not discrete. Then for any $a \in \pi(M)_{+}$we have

$$
T(a)=\left(\sup _{\varphi \ll \delta} \varphi(e)\right) \cdot a=\infty \cdot a
$$

Hence the restriction of $T$ to $\pi(M)_{+}$is completely infinite. 


\section{REFERENCES}

1. F. Combes, Poids sur une C*-algèbre, J. Math. Pures Appl. 47 (1968), 57-100.

2. F. Combes, Poids associé à une algèbre Hilbertienne à gauche, Compositio Math. 23 (1971), 49-77.

3. A. Connes, Une classification de facteurs de type III, Ann. Sci. École Norm. Sup. 4 série, 6 (1973), 133-252.

4. T. Digernes, Duality for weights on covariant systems and its applications, (Thesis), University of California, Los Angeles, 1975.

5. J. Diximier, Les $\mathrm{C}^{*}$-algèbres et leurs représentations (Cahier Scientifique 24), GauthierVillars, Paris, 1964.

6. P. Eymard, L'algèbre de Fourier d'une groupe localement compacte, Bull. Soc. Math. France 92 (1964), 181-236.

7. U. Haagerup, Operator valued weights in von Neumann algebras, To appear in J. Functional Analysis.

8. U. Haagerup, On the dual weights for crossed products of von Neumann algebras I, Math. Scand. 43 (1978), 99-118.

9. M. Landstad, Duality for covariant systems, preprint, Trondheim, 1974.

10. W. Rudin, Fourier analysis on groups (Interscience Tracts in Pure and Applied Mathematics 12), Interscience Publishers, New York - London, 1960.

11. J. Sauvageot, Sur le type de produit croisé d'une algèbre de von Neumann par une groupe localement compacte d'automorphisms, C.R. Acad. Sci. Paris, Sér. A 278 (1974), 941-944.

12. M. Takesaki, Tomitas Theory of modular Hilbert algebras and its applications, Lecture Notes in Mathematics 128), Springer-Verlag, Berlin - Heidelberg - New York, 1970.

13. M. Takesaki, The theory of operator algebras, Lecture notes, University of California, Los Angeles, 1969.

14. M. Takesaki, Duality for crossed products and the structure of von Neumann algebras of type III, Acta Math. 131 (1973), 249-310.

UNIVERSITY OF ODENSE, DENMARK 